\title{
Turn back crime: medicamentos ilegales y papel del farmacéutico comunitario
}

\author{
Mónica Andreu-March ${ }^{1,5}$, Félix Fernández-Pellicerr,5, Pilar Modamio 3,5, Eduardo L. Mariño ${ }^{4,5}$ \\ 1. Farmacéutica comunitaria. Estudiante del máster 'Medicamentos, Salud y Sistema Sanitario.' 2. Veterinario. Estudiante del máster 'Medicamentos, \\ Salud y Sistema Sanitario'. 3. Profesora titular de Farmacia Clínica y Farmacoterapia. 4. Catedrático de Farmacia Galénica. 5. Unidad de Farmacia Clínica y \\ Farmacoterapia. Departamento de Farmacia y Tecnología Farmacéutica y Fisicoquímica. Facultat de Farmàcia i Ciències de l'Alimentació. Universitat de Barcelona.
}

\section{PALABRAS CLAVE}

Productos de venta ilícita, medicamentos falsificados, complementos alimenticios, farmacia comunitaria, salud pública, promoción de la salud, credibilidad del sitio web

\section{ABREVIATURAS \\ BMPA: $\beta$-metilfeniletilamina DMAA: 1,3 \\ dimetilamilamina \\ AEMPS: Agencia Española de Medicamentos y \\ Productos Sanitarios \\ FDA: Foods and Drugs \\ Administratiosn \\ NCF: Normas de Correcta \\ Fabricación \\ OMS: Organización Mundial \\ de la Salud \\ SEVEM: Sistema Español \\ de Verificación de \\ Medicamentos \\ VIH: virus de la \\ inmunodeficiencia humana}

\section{KEYWORDS}

Illicit products, counterfeit medicines, dietary supplements, community pharmacy, public health, health promotion, website credibility

\section{RESUMEN}

El objetivo del presente trabajo fue describir y analizar la situación en España de los productos de venta ilícita, tanto medicamentos como complementos alimenticios, poniendo en conocimiento las principales acciones realizadas en los últimos meses y su contextualización a nivel mundial. Asimismo, servir de alerta sobre la magnitud del problema y la importancia del papel del farmacéutico comunitario.

Se realizó una búsqueda de información tanto a nivel nacional como internacional y se realizó un análisis descriptivo de las Notas Informativas de la Agencia Española de Medicamentos y Productos Sanitarios (AEMPS) (medicamentos ilegales, uso humano, 2015).

Los productos de venta ilícita representan un problema grave para la salud pública que no ha parado de aumentar. En España, en 2015 se publicaron 20 notas donde se prohibió y retiró un total de 35 productos. Dichos productos estaban comercializados como complemento alimenticio y presentaban un principio activo en cantidad suficiente para conferirle legalmente la condición de medicamento. El 86\% se dirigía a la disfunción eréctil.

Para minimizar este problema, es imprescindible la colaboración internacional, siendo la última y más importante operación PANGEA VIII, en junio del 2015, implicando a 115 países. En enero del 2016 entró en vigor la Convención Medicrime, tratado internacional sobre la falsificación de productos médicos y delitos similares. Por otro lado, también es necesario concienciar a la ciudadanía y dotarla de mecanismos para identificar el riesgo potencial. Los productos adquiridos fuera de los canales legales de distribución no aseguran el cumplimiento de las normas de correcta fabricación, distribución, ni posterior conservación y carecen de la información adecuada.

Turn back crime: illicit products and the role of the community pharmacist

\section{ABSTRACT}

The objective of this article was to describe and analyze the situation of illicit products in Spain, both medicines and dietary supplements, notifying main actions taken in recent months and their worldwide contextualization. Also serve as a warning about the magnitude of the problem and the importance of the role of the community pharmacist.

Searches of information were looked at a national and international level. A descriptive analysis of the Information Notes published by the Spanish Agency of Medicines and Medical Devices (AEMPS, Illegal Drugs, Human Use, 2015) was also conducted.

Illicit sale products constitute a major problem for public health that has not stopped increasing. Specifically, in Spain, 20 Notes were published in 2015. These included the prohibition and withdrawal of a total of 35 products. Marketed as dietary supplement, they contained an active ingredient enough to be legally considered as a medicine. $86 \%$ were aimed at treating erectile dysfunction.

It is essential international collaboration to minimize this issue. The last and most important operation was PANGEA VIII in June 2015, which involved 115 countries. In January 2016, the "Medicrime Convention" entered into force, an international agreement on counterfeiting of medical products and similar crimes. In addition, it is necessary to raise awareness and provide the citizens with mechanisms to identify potential risks inherent to the illicit sale products. Products purchased outside the permitted distribution channels do not ensure compliance with good manufacturing practice, distribution or subsequent storage, and they lack of adequate information.

Una parte inicial del presente artículo fue presentada como comunicación en formato póster en el VII Congreso Nacional de Farmacéuticos Comunitarios, organizado por SEFAC y celebrado en Zaragoza en mayo de 2016.

Recibido: $8 / 8 / 2016$ Aceptado: $28 / 11 / 2016$

Disponible online: $30-12-2016$

\section{Financiación: Ninguna.}

Conflicto de intereses: Ninguno.

Contribución a la autoría: PM partició en el diseño del estudio, el análisis de los resultados, la redacción del manuscrito y la revisión de contenido final. MA-M en el desarrollo de la investigación, el análisis de los resultados, la redacción del manuscrito y la revisión del contenido final. FP-C en el desarrollo de la investigación, el análisis de los resultados, la redacción del manuscrito y la revisión del contenido final. ELM en el diseño del estudio, el análisis de los resultados, la redacción del manuscrito y la revisión del contenido final.

Cite este artículo como: Andreu-March M, Fernández-Pellicer F, Modamio P, Mariño EL. Turn back crime: medicamentos ilegales y papel del farmacéutico comunitario. Farmacéuticos Comunitarios. 2016 Dec 30; 8(4):26-33. doi:10.5672/FC.2173-9218.(2016/Nol8).004.04 Correspondencia: Pilar Modamio (pmodamio@ub.edu)

ISSN 1885-8619 @SEFAC (Sociedad Española de Farmacia Familiar y Comunitaria). Todos los derechos reservados. 


\section{Introducción}

En la actualidad, existe un considerable mercado ilegal de productos con principios activos incluidos donde un ciudadano "mal informado" y seducido por las indicaciones, la apariencia de complemento alimenticio, la etiqueta de "producto natural" y su fácil accesibilidad a través de Internet, puede, entre otros motivos, optar por su adquisición sin pensar en los efectos indeseados, a menudo irreversibles. Este problema se puede agravar en individuos con comorbilidades para los que determinados medicamentos de prescripción están contraindicados y como consecuencia recurren a menudo a buscar alternativas [1-4].

El farmacéutico comunitario, como profesional de la salud, tiene la responsabilidad de promover el uso racional del medicamento. Debe, por lo tanto, concienciar e informar a la ciudadanía del peligro que supone para su salud la adquisición de "remedios" (ya sean medicamentos o complementos alimenticios) a través de vías ilícitas, así como la importancia de comunicar cualquier sospecha de un efecto imprevisto y/o indeseado. Para todo ello es imprescindible la formación continua y la colaboración con las Administraciones sanitarias y los demás profesionales de la salud [4-7].

Durante el año 2014, Turn back crime ("Acabemos con la delincuencia") dio nombre a la campaña mundial de concienciación emitida por la Interpol que pretendía poner de manifiesto el alcance del crimen organizado y sensibilizar a los ciudadanos sobre los peligros que plantean, dentro de los medicamentos ilegales, los medicamentos falsificados, proporcionando además la información necesaria para poder identificarlos [8].

El objetivo del presente trabajo fue, en primer lugar, describir y analizar la situación de los productos de venta ilícita en España, tanto medicamentos como complementos alimenticios, siendo necesario para ello conocer las principales acciones que se han llevado a cabo en los últimos meses y su contextualización a nivel mundial. En segundo lugar pretende servir de alerta sobre la magnitud del problema y la importancia del papel del farmacéutico comunitario.

\section{Método}

Se realizó una revisión bibliográfica de los principales artículos de revisión, notas informativas y noticias, mayoritariamente del último año 2015, relacionados con el tráfico ilícito de medicamentos y complementos alimenticios.

Se comprobó que se trataba de una problemática global, imposible de aislar en un único país, por lo que fue necesario ampliar la búsqueda y situarla tanto a nivel nacional como internacional. De este modo, las fuentes de información que se constituyeron en el pilar básico de la revisión fueron las sedes web de organizaciones internacionales como la Organización Mundial de la Salud (OMS) y la Interpol (responsable de la coordinación de operaciones internacionales para combatir delitos farmacológicos). A nivel del estado español, la información de referencia provino de la Agencia Española de Medicamentos y Productos Sanitarios (AEMPS), y se realizó además un análisis descriptivo de todas las notas informativas publicadas en su sede web, correspondiente a la categoría medicamentos de uso humano, medicamentos ilegales, del año 2015.

Las palabras clave empleadas en la búsqueda de información fueron: counterfeit medicines, illicit pharmaceuticals, undeclared drugs, Pangea, Medicrime, medicamentos ilegales.

Los resultados y discusión se organizaron por apartados, siguiendo el siguiente esquema: situación global, situación en España, operaciones realizadas, un ejemplo concreto (estimulantes anfetamínicos en complementos alimenticios) y proyectos $\mathrm{y}$ estrategias.

\section{Resultados y discusión}

Siguiendo el esquema anteriormente indicado y su expresa mención de alguno de los resultados obtenidos, podemos señalar lo siguiente.

\section{Situación global}

La diversidad de la terminología empleada, la complejidad legislativa y una organización sofisticada, dificultan la estimación de los daños producidos por los productos de ven- ta ilícita. Sin embargo, es bien conocido que el número de productos que entran de manera ilegal en la cadena de distribución es un problema grave para la salud pública que no ha dejado de aumentar en los últimos años [9,10]. Así, en 2010 se asoció a la venta de productos ilegales, incluidos los medicamentos falsificados, 75.000 millones de dólares únicamente en Estados Unidos, lo que supondría un aumento del 90\% respecto al 2005 [1].

La fabricación y distribución de este tipo de productos se ve favorecida por la existencia de un elevado número de usuarios potenciales (pacientes en muchos casos). Por otro lado, tampoco supone un elevado coste por parte de los falsificadores, ya que no siguen las directrices de las Normas de Correcta Fabricación (NCF), omiten o, en su lugar, emplean ingredientes más baratos $\mathrm{y}$ no requieren de una infraestructura sofisticada. Además, en muchas ocasiones los productos son directamente sustraídos a sus fabricantes originales, para ser, a continuación, manipulados o almacenados incorrectamente, reetiquetados para cambiar la fecha de vencimiento y finalmente revendidos a precios más bajos. En su distribución se suelen utilizar los canales convencionales de comercio internacional $[10,11]$.

Sin embargo, el incremento de su utilización se debe, en gran parte, a la venta por Internet. Según la OMS, más del 50\% de los medicamentos comprados a través de la red en páginas que ocultan su dirección física, son ilegales. Además, en la red se adquieren un gran número de complementos alimenticios, entre los que destacan adelgazantes adulterados y aquellos relacionados con la disfunción eréctil. Sin embargo, también circulan un gran número de medicamentos de prescripción, tanto genéricos como medicamentos de marca, muchos destinados cada vez más a tratar enfermedades graves, como el cáncer, antirretrovirales para el virus de la inmunodeficiencia humana (VIH) o antibióticos. Son precisamente los antibióticos y los antipalúdicos los notificados con más frecuencia a la OMS. Se ha cuantificado que sólo en África mueren aproximadamente 100.000 personas al año como consecuencia de medicamentos falsos contra la malaria $[1,2,9,11]$. 


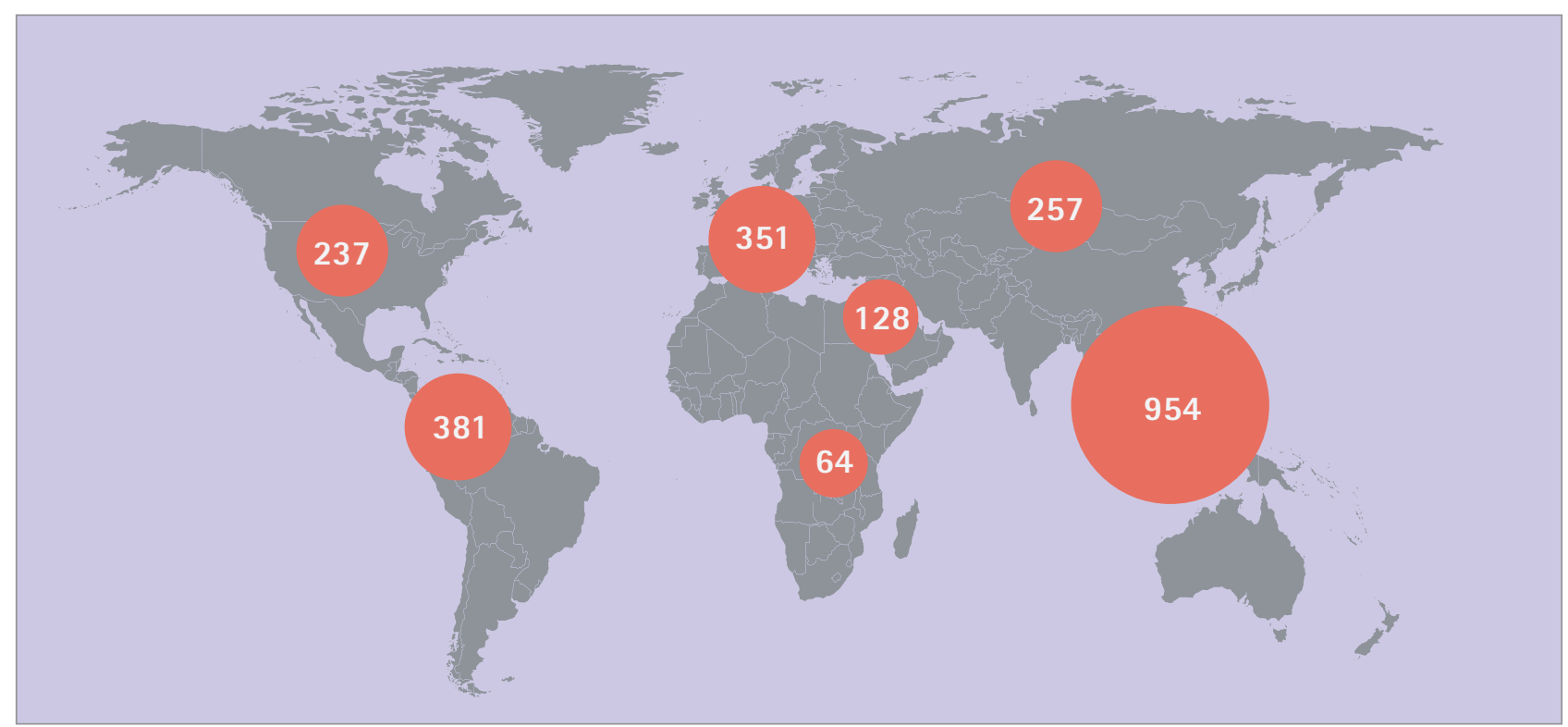

Figura 1 Distribución geográfica de los incidentes por delitos farmacéuticos en 2011. (Adaptación de la referencia número 11)

En la figura 1 se puede observar la distribución geográfica de los incidentes por delitos farmacéuticos en 2011 [11]. Cabe destacar la dificultad que existe a la hora de comparar las diferentes regiones. Aquellas con una cifra más elevada de delitos relacionados con productos de venta ilícita corresponden a una mayor notificación de los casos, que suele significar que estos países están identificando de forma efectiva este tipo de delincuencia mediante inspecciones y aplicación de la ley. Esto implica, por lo tanto, que las regiones más frecuentemente vinculadas a los incidentes no siempre son aquellas asociadas a programas de control e inspección más frágiles $[10,11]$.

\section{Situación en España}

En España, concretamente el pasado año 2015, la AEMPS publicó 20 notas informativas en las que se procedía, como medida cautelar, a la prohibición y retirada del mercado de todos los ejemplares de un total de 35 productos. Éstos, principalmente, incluían un principio activo (notificado o no a las autoridades) en cantidad suficiente para conferirle legalmente la condición de medicamento, aunque no figuraba en el etiquetado y se encontraban además comercializados como complemento alimenticio (figura 2). Otro aspecto destacable es que el $74 \%$ se había fabricado en España. Por otro lado, se observó que en su mayoría se destinaban al tratamiento de la disfunción eréctil

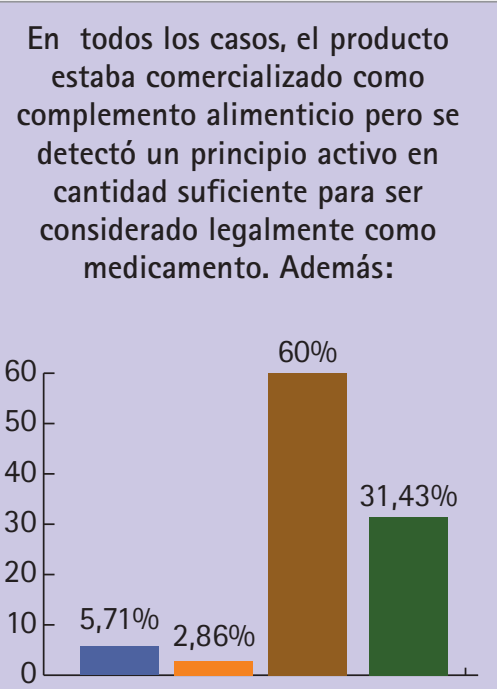

El producto no fue notificado por la empresa a las autoridades competentes

El producto no fue notificado por la empresa a las autoridades competentes. En el etiquetado se declaró un principio activo que no se correspondió con el detectado

El producto no fue notificado por la empresa a las autoridades competentes. El principio activo no fue declarado en el etiquetado

El principio activo no fue declarado en el etiquetado

Figura 2 Descripción de los distintos motivos de retirada de los productos. (Notas informativas de medicamentos de uso humano/medicamentos ilegales/2015)

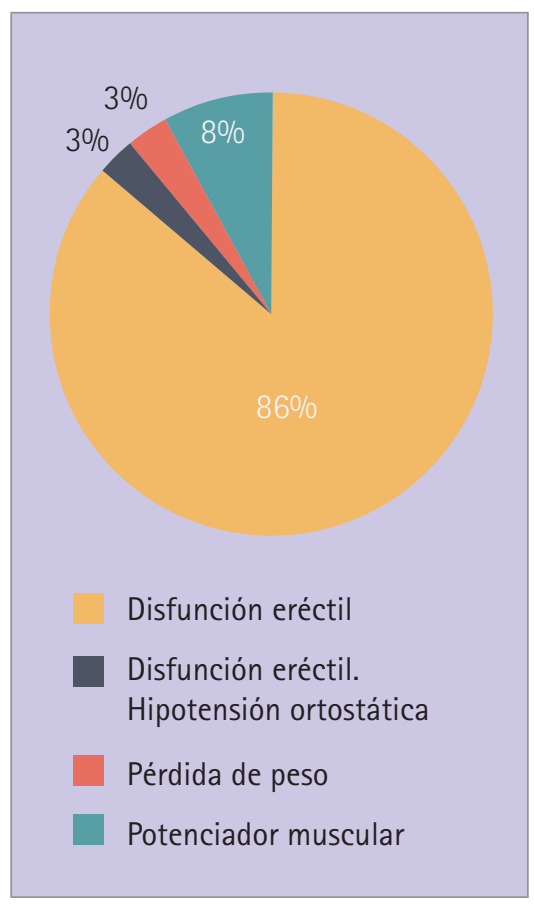

Figura 3 Indicaciones de los productos retirados. (Notas informativas de medicamentos de uso humano/ medicamentos ilegales/2015)

y, en menor medida, al desarrollo muscular y a la pérdida de peso (figura 3). De este hecho derivaba que los principios activos no declarados más frecuentemente fueran sildenafilo y sus derivados (figura 4). Es importante recordar que los inhibidores selectivos de la fosfodiesterasa 5 están contraindicados en personas con factores de riesgo cardiovascular. Por otro lado, los relacionados con la testosterona están asociados a hepatotoxicidad grave, insuficiencia renal y 


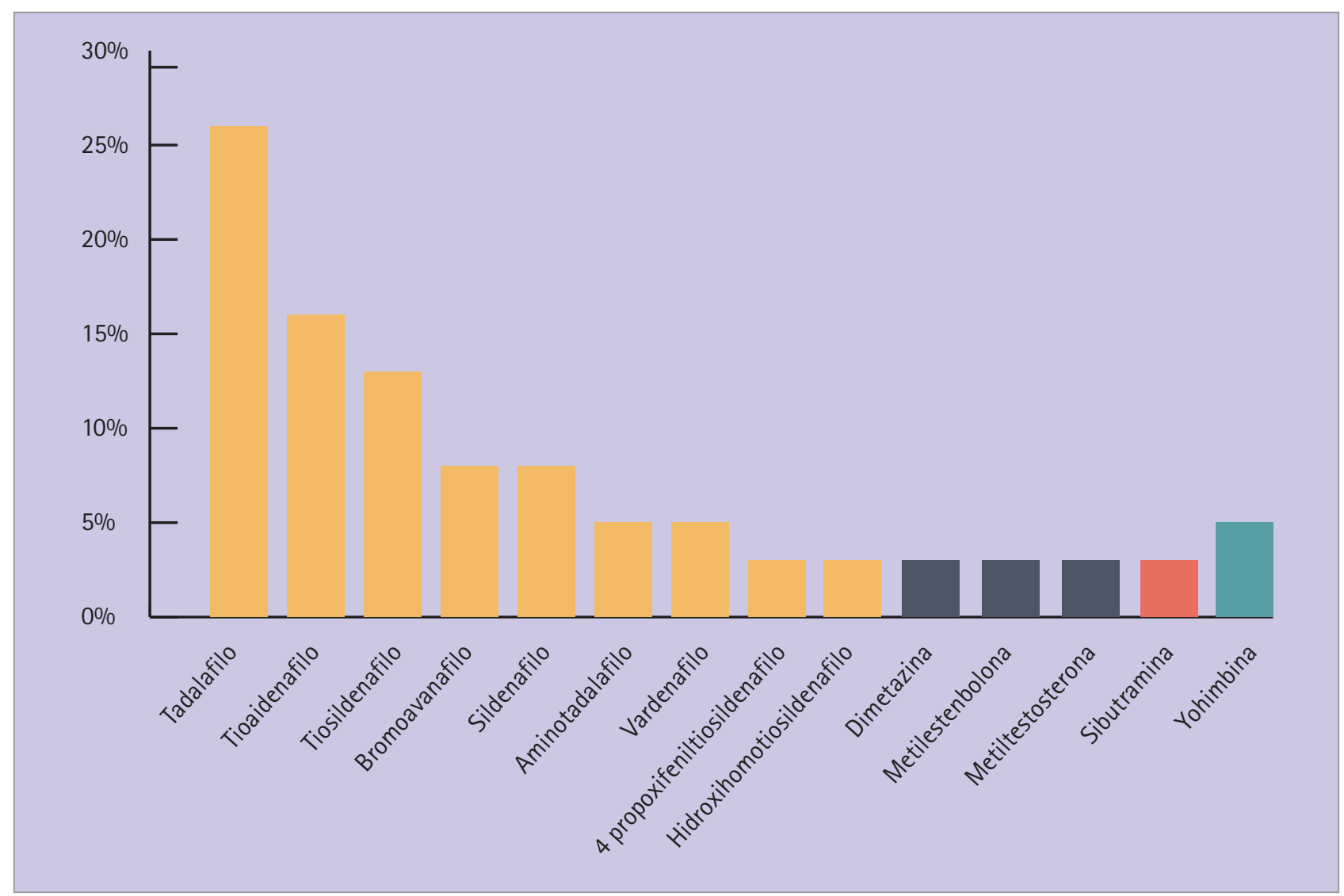

Figura 4 Principios activos más frecuentemente empleados de forma fraudulenta. (Notas Informativas de medicamentos de uso humano/ medicamentos ilegales/2015)

embolia pulmonar y aquellos relacionados con anfetamina, como sibutramina, pueden provocar accidentes vasculares. Además, hay que destacar que todos ellos interaccionan con un gran número de medicamentos [2,4].

Más recientemente, en concreto, el pasado 5 de abril de 2016, la AEMPS emitió una nota informativa advirtiendo de una nueva realidad: su actuación durante los últimos meses frente a la venta ilegal de productos a través de plataformas de anuncios clasificados (tanto a través de Internet como en aplicaciones móviles). Se trata de plataformas de compraventa entre particulares que no disponen de la autorización legal de venta de medicamentos, por lo que carecen de la información necesaria, no garantizan su calidad ni su correcta supervisión. Muchos de ellos además son medicamentos que requieren prescripción médica, como Bexsero ${ }^{\circledR}$ (vacuna contra la meningitis B que ha sufrido problemas de suministro en las farmacias) a través de varias aplicaciones $[12,13]$.

\section{Operaciones realizadas}

Es importante destacar, tal y como se ha comentado, que se tra- ta de un problema a nivel mundial. Durante 2011 fueron incautados unos 30 millones de medicamentos falsificados únicamente en las fronteras europeas. Por este motivo es imprescindible la colaboración internacional de los diferentes organismos y empresas privadas, así como la realización de grandes operaciones coordinadas por la Interpol. La última $\mathrm{y}$ más importante hasta la fecha fue la denominada PANGEA VIII, llevada a cabo en junio de 2015. Dicha operación implicó a 115 países y fue la responsable del cierre de 2.414 sitios web, la detención de 156 personas y la incautación de 20,7 millones de productos $[11,14,15]$. En la tabla 1 se detalla información sobre las operaciones PANGEA II a VIII, de 2009 a 2015, respectivamente [14].

En nuestro país, durante PANGEA VIII la Guardia Civil incautó 1.022.352 dosis, de las cuales 285.269 estaban dirigidas al tratamiento de la disfunción eréctil, 235.424 al desarrollo de la masa muscular, 118.627 psicoestimulantes, 40.206 suplementos vitamínicos, 40.122 analgésicos y antibióticos, y 790 productos para la pérdida de peso. Además, se produjeron 837 inspecciones en centros deportivos, sex shops, tiendas de alimentos saludables, así como también en herbolarios y farmacias. Concluyó con el registro de 196 infracciones administrativas, 4 delitos y la detención de 19 personas [14].

En España también se han llevado a cabo otras operaciones desarrolladas de forma conjunta entre las diferentes comunidades autónomas $\mathrm{y}$ la AEMPS, que en el último período 2012-1015 (incluyendo PANGEA) culminaron en más de 10.000 controles a la importación de principios activos por parte de las delegaciones y subdelegaciones de Gobierno, unas 400 inspecciones para verificar el cumplimiento de buenas prácticas en la distribución, y la investigación de más de 1.100 webs de venta ilegal de medicamentos, tanto de uso humano como veterinario [16].

\section{Un ejemplo instructivo}

de los resultados encontrados: estimulantes anfetamínicos en complementos alimenticios

Los complementos alimenticios que incorporan sustancias estimulantes se han extendido por todo el mundo [3,17]. Un ejemplo fue la detección 
Tabla 1 Operaciones Pangea II a VIII, de 2009 a 2015 respectivamente, según: fecha, países participantes, número de sitios webs cerrados, productos incautados, arrestos. (Adaptación de la referencia número 14)

\begin{tabular}{|c|c|c|c|c|c|c|c|}
\hline & \multicolumn{7}{|c|}{ Pangea } \\
\hline & II & III & IV & V & VI & VII & VIII \\
\hline Fecha & $\begin{array}{c}16-20 \text { Nov } \\
2009\end{array}$ & $\begin{array}{c}5-12 \text { Oct } \\
2010\end{array}$ & $\begin{array}{c}\text { 20-27 Sep } \\
2011\end{array}$ & $\begin{array}{c}25 \text { Sep-2 Oct } \\
2012\end{array}$ & $\begin{array}{c}\text { 18-27 Jun } \\
2013\end{array}$ & $\begin{array}{c}\text { 13-20 May } \\
2014\end{array}$ & $\begin{array}{c}9-16 \text { Jun } \\
2015\end{array}$ \\
\hline Paises participantes & 25 & 44 & 81 & 100 & 99 & 111 & 115 \\
\hline Sitios web cerrados & 153 & 297 & 13.500 & $>18.000$ & 13.700 & 10.600 & 2.414 \\
\hline Productos incautados & 167.000 & $>2.000 .000$ & 2.400 .000 & 3.750 .000 & 9.800 .000 & 9.400 .000 & 20.700 .000 \\
\hline Arrestos & 59 & 87 & 92 & 80 & 213 & 239 & 156 \\
\hline
\end{tabular}

de adulteración del suplemento para

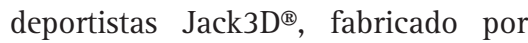
UspLabs. En 2011, la Foods and Drugs Administratiosn (FDA) prohibió su comercialización en los Estados Unidos por contener 1,3 dimetilamilamina (DMAA), un derivado anfetamínico. Sin embargo, el laboratorio desvió su comercialización hacia Europa, hasta que en octubre del mismo año Dinamarca denunció la presencia de DMAA en el producto. La alerta se extendió a nivel mundial, debido a su distribución vía Internet mediante diferentes sociedades con sede en Portugal, Eslovenia y Reino Unido.

$\mathrm{Al}$ cabo de un tiempo, se produjo una situación similar, al retirarse del mercado por adulteración otro suplemento de UspLabs, OxyeLitePro ${ }^{\circledR}$. En esta ocasión, se reintrodujo en 2013 sustituyendo el DMAA por otra sustancia. Sin embargo, en 2014 se habían declarado hasta 97 casos de hepatitis aguda no viral (incluidos 3 trasplantes de hígado y una muerte) en consumidores habituales del producto en los Estados Unidos. La FDA detectó entonces que el laboratorio había utilizado aegelina sin comunicar la seguridad de la misma, y ordenó su retirada del mercado [18-21].

Desafortunadamente, no se trata de una situación aislada. La FDA advirtió a otras cinco empresas que retiraran complementos alimenticios anunciados como naturales por contener Acacia rigidula, en los que se detectó un isómero de las anfetaminas nunca antes autorizado, la $\beta$-metilfeniletilamina (BMPA). Pasado más de un año, Cohen et al. publicaron en el año 2016 que tras analizar 21 de las marcas afecta- das, 11 aún contenían BMPA, e "invitando" a la FDA a adoptar acciones más contundentes para eliminar este estimulante del mercado [22].

En noviembre de 2015, el Departamento de Justicia americano y seis Agencias Federales iniciaron causas civiles y penales contra UspLabs $\mathrm{y}$ otros fabricantes y distribuidores de complementos alimenticios, por importar y utilizar ingredientes con falsos certificados de análisis y falsas etiquetas [26].

Este ejemplo entrevé la complejidad de un conflicto que se hace mayor debido a la distribución por la red. El actual modelo de comercio de complementos alimenticios implica un desafío a nivel mundial: la necesidad de disponer de un sistema capaz de detectar aquellos que presentan efectos adversos antes de que lleguen a los potenciales consumidores, hecho que debería ser garantizado y avalado por una ley [17].

\section{Proyectos y estrategias}

A raíz de esta nueva realidad, el 1 de enero del 2016 entró en vigor la denominada Convención Medicrime, tratado internacional patrocinado por el Consejo de Europa sobre la falsificación de productos médicos y delitos similares que supongan una amenaza para la salud pública, y que incluye ya a 23 países de Europa, África y Asia. Su principal novedad es la tipificación en el Código Penal de nuevos delitos que comporten además un incremento de las penas [27,28]. Por otro lado, dando respuesta a los planteamientos de la Directiva Eu- ropea, España dispone de tres años (2016-19) para la creación y puesta en marcha del Sistema Español de Verificación de Medicamentos (SEVEM). Este sistema comportará la inclusión por parte del fabricante de dos dispositivos de seguridad en los medicamentos, en concreto, un identificador único y un dispositivo contra la manipulación, que permitirá a los distribuidores y a las farmacias comunitarias identificar individualmente los envases y verificar su autenticidad. Los medicamentos afectados, en su mayoría de prescripción, serán publicados en una lista por la AEMPS $[29,30]$. En la figura 5 se muestra el detalle de verificación en el punto de dispensación del indicado SEVEM [29].

No obstante, se ha demostrado que una de las medidas más efectivas en el ámbito de la salud pública es concienciar a la ciudadanía y dotarla de mecanismos para que pueda identificar un riesgo potencial para su salud. En esta línea, la AEMPS elaboró una Estrategia frente a medicamentos falsificados, y recién ha publicado la tercera edición 2016-19. Esta estrategia incluye diferentes campañas informativas dirigidas a la ciudadanía y la recomendación de asegurarse siempre de que el canal de venta on-line sea legal, ya que Internet se ha establecido como la principal vía de adquisición de productos de venta ilícita en nuestro país [31-33]. Las figuras 6 y 7 representan, respectivamente, una compilación de los principales consejos emitidos por parte de diferentes instituciones en sus campañas de concienciación; y una 


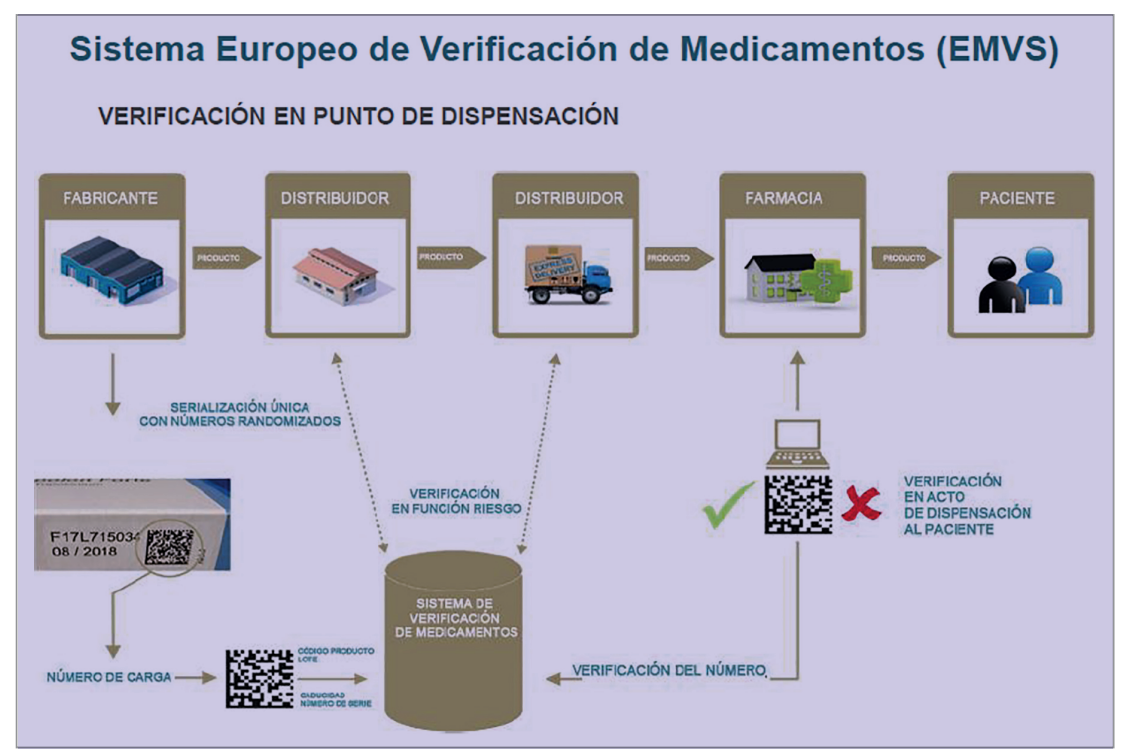

Figura 5 Sistema Europeo de Verificación de Medicamentos: verificación en punto de dispensación [29].

Para saber si estoy ante un medicamento ilegal... ¿Qué debe hacerme sospechar?

\begin{tabular}{|l|l|}
\hline En la web: & Ante el producto: \\
\hline - Sin logotipo o certificado de veri- & - Errores ortográficos y gramatica- \\
ficación (figura 7) & les en el envase o prospecto. \\
- Sin la información mínima requeri- & - El medicamento no tiene el aspec- \\
da sobre los responsables de la pá- & to, tacto y olor que debería. \\
gina, ubicación o datos de contacto. & - No coinciden los números de lote \\
- Inducen a error sobre el lugar de & y fecha de caducidad del envase \\
procedencia de los medicamentos. & primario (interior) y secundario \\
- No aseguran adecuadamente la & (exterior). \\
protección de los datos personales. & - El envase no está en buenas con- \\
- Ofrece productos a precios sospe- & diciones. \\
chosamente bajos y/o a través de & - Contiene un prospecto en distin- \\
anuncios por correo electrónico o & to idioma que el utilizado en el \\
aplicaciones móviles. & anuncio. \\
- Ofrece medicamentos que requie- & - El efecto del medicamento no es \\
ren receta médica, cosa totalmen- & el esperado o provoca alguna re- \\
te prohibida en España. & acción no habitual. \\
\hline
\end{tabular}

Ante cualquier duda o sospecha, consultar siempre a un profesional sanitario

Figura 6 Principales consejos emitidos por parte de diferentes instituciones en sus campañas de concienciación $[5,6,34]$

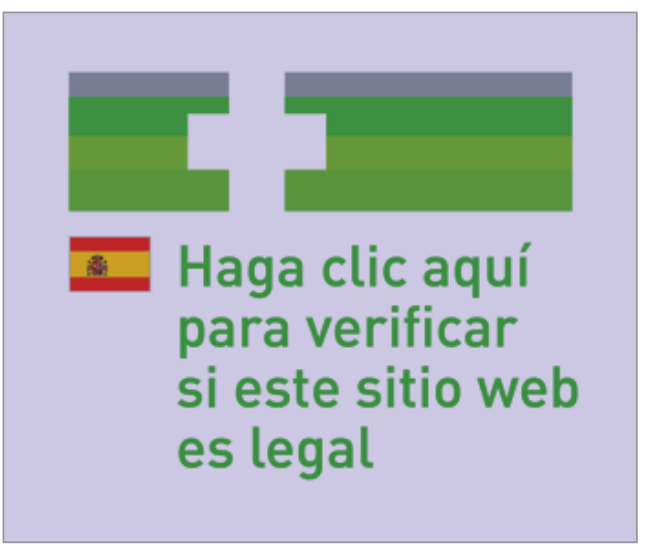

Figura 7

Logotipo común diseñado por la Comisión Europea. Permite a los usuarios identificar el Estado Miembro en el que está establecida la persona que ofrece medicamentos por venta a distancia, y reconocer los sitios webs de las farmacias que dispensan legalmente a través de Internet $[6,9,34]$ imagen del logotipo común diseñado por la Comisión Europea que permite a los usuarios identificar el Estado Miembro y reconocer los sitios webs de las farmacias que dispensan legalmente a través de Internet [5,6,9,34].

En este sentido, los farmacéuticos comunitarios disponen de una posición privilegiada por su conocimiento sobre el medicamento y la cercanía ante sus pacientes/usuarios, para convertirse en un adecuado canal de información y actuar como nexo de unión con las Administraciones. Desde las farmacias comunitarias podría difundirse información impresa (dipticos, etc.) y/o material audiovisual (vídeos, etc.) que engloben los principales consejos y recomendaciones emitidos, de forma especial en la red y aplicaciones móviles, así como recomendar páginas web oficiales e informativas, promoviendo el empoderamiento del paciente. Destaca la web del proyecto europeo Fakeshare, al que se ha sumado activamente la AEMPS, donde tanto el profesional sanitario como los ciudadanos pueden, de manera fácil y sencilla, comprobar sitios web de venta de medicamentos, verificar productos ilegales conocidos, denunciar una farmacia on-line o un producto, así como consultar noticias relacionadas, entre otros [35]. También la AEMPS dispone de un sitio web, Distafarma, centrado en la venta a distancia, y donde se pueden encontrar y descargar el tríptico y póster de la campaña informativa (figura 8) [36].

Otro aspecto importante destacable con relación al farmacéutico es que tiene la capacidad de identificar aquellos pacientes susceptibles, donde es de mayor prioridad alertarles y centrar su atención de forma personalizada, o bien mediante la organización de reuniones en grupo. Asimismo, sería interesante combinarlo con alguno de los materiales informativos antes citados, orientados a un perfil concreto de paciente, como por ejemplo aquellos que demandan potenciadores de la actividad sexual, suplementos para deportistas que pueden estar adulterados o bien anorexígenos.

Cabe destacar que durante el último congreso Infarma 2016 celebrado en Madrid, la Jefa del Departamento de Inspección de la AEMPS, anunció ya la nueva estrategia de información por parte de la agencia española, en 


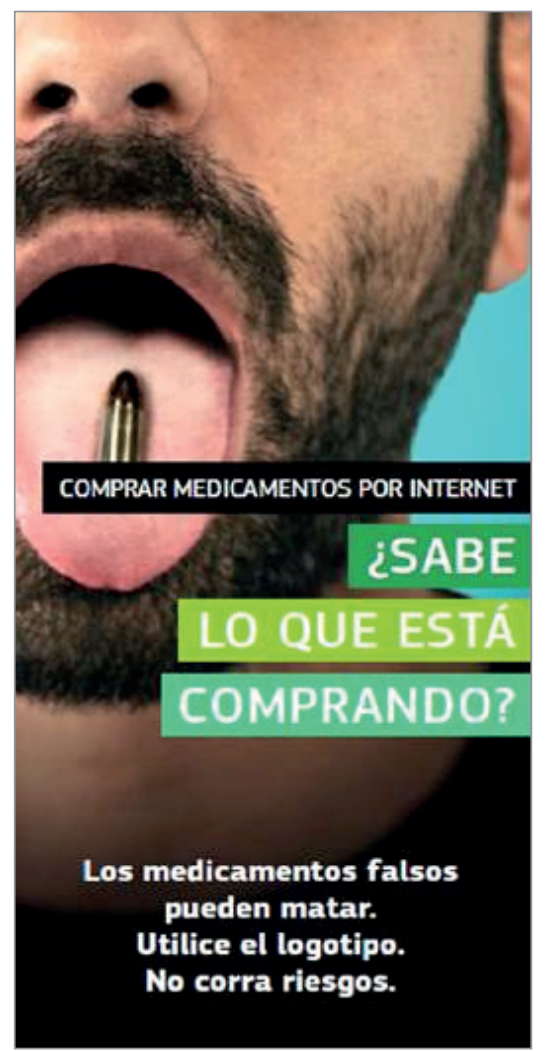

Figura 8 Póster correspondiente a la campaña institucional de venta a distancia, a través de Internet, de medicamentos de uso humano no sujetos a prescripción médica [36].

coordinación con otras agencias europeas, centrada en el uso de las redes sociales. También destacó el poder del buscador Google con el fin de priorizar las webs legales. Finalmente, declaró: "El farmacéutico juega un papel clave para que no lleguen medicamentos falsificados a los pacientes" [32,37].

\section{Conclusiones}

Los productos de venta ilícita se presentan como una opción cada vez más accesible ante el usuario, con consecuencias para su salud potencialmente graves. Afecta a todas las regiones del mundo y abarca tanto complementos alimenticios como medicamentos de prescripción. Pueden no presentar eficacia por falta de principios activos o, por el contrario, efectos indeseados por estar en exceso o no declarados, además de otras adulteraciones. Asimismo, aquellos productos adquiridos fuera de los canales legales de distribución no aseguran el cumplimiento de las NCF, distribución, ni su posterior conservación, y carecen de la información adecuada. Estos hechos han ocasio- nado, en los últimos años, la necesidad de adoptar y coordinar diferentes medidas tanto a nivel nacional como internacional. Es imprescindible, simultáneamente, concienciar a la ciudadanía y proporcionarle los medios necesarios para poder evitar e identificar un posible fraude (empoderamiento del paciente). Sobre el farmacéutico comunitario recae la responsabilidad de garantizar una atención de calidad tanto en la dispensación como en su labor formativa/informativa.

\section{Referencias bibliográficas}

1. OMS. La falsificación de medicamentos: una amenaza creciente. [Internet]. World Health Organization. 2010 [Acceso 8/4/2016]. Boletín OMS; 88:241-320. Disponible en: http://www.who.int/bulletin/volumes/88/4/10-020410/es/

2. Agencia Española de Medicamentos y Productos Sanitarios. Notas informativas de medicamentos de uso humano/medicamentos ilegales/2015 [Internet]. 2015. Disponible en: http:// www.aemps.gob.es/informa/notasInformativas/medicamentosUsoHumano/medIlegales/2015/home.htm

3. Rocha T, Amaral JS, Oliveira MBPP. Adulteration of Dietary Supplements by the Illegal Addition of Synthetic Drugs: A Review. Compr Rev Food Sci Food Saf [Internet]. 2016 Jan;15(1):43-62. [Acceso 8/4/2016]. Disponible en: http://doi.wiley. com/10.1111/1541-4337.12173

4. Andreu March M, Fernández Pellicer F, Viñas Bastart M, Domínguez Vela C, Alban Robayo M, Cruz Guaman G, et al. Análisis de las comunicaciones sobre medicamentos ilegales publicadas en la agencia Española de Medicamentos y Productos Sanitarios: punta del iceberg a considerar por el farmacéutico comunitario. Farm Comunitarios [Internet]. 2016;8(Suplemento 1). Disponible en: http:// www.farmaceuticoscomunitarios.org/ es/journal-article/analisis-comunicaciones-sobre-medicamentos-ilegales-publicadas-agencia-espanola

5. Organización Mundial de la Salud. OMS | Productos médicos de calidad subestándar, espurios, de etiquetado engañoso, falsificados o de imitación (SSFFC) [Internet]. Centro de Prensa. World Health Organization; 2016 [cited 2016 Mar 29]. Disponible en: http://www.who.int/mediacentre/ factsheets/fs275/es/

6. Generalitat de Catalunya. Els medicaments il-legals [Internet]. Canal salut. Medicaments i farmàcia. 2016. Dispo- nible en: http://medicaments.gencat. cat/ca/ciutadania/informacio-i-consells/preguntes-frequents/medicaments-per-internet/

7. Abellán-García Sánchez F, Baixauli Fernández VJ, Celada Carrizo A, Gastelurrutia Garralda MÁ, Guitiérrez Ríos P, López Guzmán J, et al. Código Ético de la Farmacia Comunitaria [Internet]. 2015. Disponible en: https:// www.sefac.org/media/2015/codigo_ etico_sefac.pdf

8. Interpol. Turn Back Crime [Internet]. 2015. Disponible en: http://www.interpol.int/es/News-and-media/TurnBack-Crime/Turn-Back-Crime

9. European Medicines Agency. Falsified medicines [Internet]. Human regulatory. 2016. Disponible en: http:// www.ema.europa.eu/ema/index.jsp?curl=pages/special_topics/general/ general_content_000186.jsp\&tmi$\mathrm{d}=$ WCOb01ac058002d4e8

10. Pharmaceutical Security Institute. Sede Web [Internet]. 2016. Disponible en: http://www.psi-inc.org/index.cfm

11. Johns Hopkins Model United Nations Conference (JHUMUNC). Background Guide Session XVIII. Topic 2: Trafficking in Fraudulent Medicines. Baltimore, MD; February 5-8, 2015.

12. DiarioFarma. Bexsero, Varivax, Gonal, Menopur o Rotateq, a golpe de click en Wallapop. 2016 Apr 1; Disponible en: http://www.diariofarma.com/2016/04/01/bexsero-varivax-gonal-menopur-o-rotateq-a-golpe-de-click-en-wallapop?id=25224

13. Agencia Española de Medicamentos Productos Sanitarios. Nota informativa: Advertencia en relación con la venta de medicamentos a través de webs y aplicaciones para el móvil de compraventa de entre particulares. 5 de abril de 2016 [Internet]. [cited 2016 Apr 9]. Disponible en: http://www.aemps.gob.es/informa/ notasInformativas/medicamentosUsoHumano/medIlegales/2016/docs/ ICM_MI_02-2016-compraventa-particulares.pdf

14. International Institute of Research Against Counterfeit Medicines (IRACM). PANGEA VIII: over $20 \mathrm{mi}-$ llion of illegal and fake drugs seized in 115 countries. 2015 Jun 23. Disponible en: http://www.iracm.com/ en/2015/06/pangea-viii-over-20-million-of-illegal-and-fake-drugs-seized-in-115-countries/

15. Interpol. Una operación coordinada por INTERPOL contra la delincuencia organizada se salda con la incautación de 20 millones de medicamentos ilícitos. Centro de Prensa [Internet]. Lyon (Francia); 2015 Jun 18; Disponible en: http://www.interpol.int/ es/Centro-de-prensa/Noticias/2015/ N2015-082/ 
16. Ministerio de Sanidad S sociales e I. Nota de prensa: Estrategia de la Agencia Española de Medicamentos y Productos Sanitarios frente a medicamentos falsificados. [Internet]. 2016 [cited 2016 May 30]. Disponible en: http://www.aemps.gob. es/informa/notasPrensa/docs/2016/ nota-prensa_2016-04-11-investigacion-web-venta-ilegal.pdf

17. Geller AI, Shehab N, Weidle NJ, Lovegrove $\mathrm{MC}$, Wolpert $\mathrm{BJ}$, Timbo $\mathrm{BB}$, et al. Emergency Department Visits for Adverse Events Related to Dietary Supplements. N Engl J Med [Internet]. 2015 Oct 15 [cited 2016 Apr 25];373(16):1531-40. Disponible en: http://www.nejm.org/doi/ abs/10.1056/NEJMsa 1504267

18. Bucchini L, La Pira R. Stop all'integratore brucia grassi 0xy ELITE, segnalati 62 casi di epatite. Il Ministero lancia l'allerta. In Italia non è in vendita ma si compra on line. il fatto alimentare [Internet]. 2013 Nov 21; Disponible en: http://www.ilfattoalimentare.it/stop-integratore-oxy-elite-62-casi-epatite-ministero-salute-allerta.html

19. Bucchini L. Allerta europea: ritirato dal mercato l'integratore Jack3d, contiene sostanze dopanti pericolose per la salute. il fatto alimentare [Internet]. 2012 Jun 20; Disponible en: http://www.ilfattoalimentare.it/allerta-integratore-jack3d.html

20. European Comission - RASFF Portal. Notification details - 2012.0798. Unauthorised substance 1,3 dimethylamylamine (DMAA) in food supplement from the United States, via the Netherlands [Internet]. 2012. Disponible en: https://webgate.ec.europa.eu/rasff-window/portal/index. $\mathrm{cfm}$ ? event $=$ notificationDetail\&NO TIF_REFERENCE $=2012.0798$

21. U.S. Food and Drug Administration. FDA Investigation Summary: Acute Hepatitis Illnesses Linked to Certain OxyElite Pro Products [Internet]. 2014. Disponible en: http://www.fda. gov/Food/RecallsOutbreaksEmergencies/Outbreaks/ucm370849.htm

22. Cohen PA, Bloszies C, Yee C, Gerona R. An amphetamine isomer whose efficacy and safety in humans has never been studied, $\beta$-methylphenylethylamine (BMPEA), is found in multiple dietary supplements. Drug Test Anal [Internet]. 2016 Mar [cited 2016 Apr 25];8(3-4):328-33. Disponible en: http://doi.wiley.com/10.1002/dta.1793

23. Bonardi B. Integratori alimentari: in Usa causano 23.000 accessi al pronto soccorso ogni anno. Il problema principale è costituito dai disturbi cardiaci. il fatto alimentare [Internet]. 2015
Oct; Disponible en: http://www.ilfattoalimentare.it/integratori-alimentari-pronto-soccorso-usa.html

24. O'Connor A. Study Warns of Diet Supplement Dangers Kept Quiet by F.D.A. The New York Times [Internet]. 2015 Apr 7; Disponible en: http:// well.blogs.nytimes.com/2015/04/07/ study-warns-of-diet-supplementdangers-kept-quiet-by-f-d-a/

25. The Editorial Board. Conflicts of Interest at the F.D.A. The New York Times [Internet]. 2015 Apr 13; Disponible en: http://www.nytimes.com/2015/04/13/ opinion/conflicts-of-interest-at-thefda.html?_r=2

26. Department of Justice. Justice Department and Federal Partners Announce Enforcement Actions of Dietary Supplement Cases. Office of Public Affairs [Internet]. 2015 Nov 17; Disponible en: https://www.justice.gov/ opa/pr/justice-department-and-federal-partners-announce-enforcement-actions-dietary-supplement-cases

27. Agencia Española de Medicamentos y Productos Sanitarios. Nota informativa: Puesta en marcha de la convención Medicrime contra la falsificación de medicamentos. Diciembre de 2015 [Internet]. [cited 2016 Apr 8]. Disponible en: http://www.aemps. gob.es/informa/notasInformativas/ medicamentosUsoHumano/medllegales/2015/docs/ICM_MI 19-15-Convencion-Medicrime.pdf

28. García M. España es pionera en la Unión Europea en combatir la falsificación de medicamentos. ElGlobal.net [Internet]. Madrid; 2015 0ct 9; Disponible en: http://www.elglobal.net/ noticias-medicamento/2015-10-09/ industria-farmaceutica/espana-es-pionera-en-la-union-europea-en-combatir-la-falsificacion-de-medicamentos/ pagina. aspx?idart=941113

29. Sanz I. Medicamentos falsificados: El sistema Europeo de Verificación de Medicamentos (EMVS) y su implementación en España. Objetivo, contenido y plazos. XII Seminario Industria Farmacéutica y Medios de Comunicación. [Internet]. Farmaindustria; 2015. Disponible en: http://www.farmaindustria.es/web/wp-content/uploads/ sites/2/2015/11/9-Iciar-Sanz-de-Madrid-Seminario-Pdtas.-2015.pdf

30. Consejo General de Colegios Oficiales de Farmacéuticos. Al Día Consejo. Hacia la creación de un Sistema Español de Verificación de Medicamentos SEVEM. Farmacéuticos [Internet]. 2015;411:18. Disponible en: http:// publicaciones.portalfarma.com/farmaceuticos/411/files/assets/basic-ht$\mathrm{ml} /$ page $18 . h t m l$
31. El Global. La Aemps intensifica el cierre de webs ilegales que venden fármacos. ElGlobal.net [Internet]. Madrid; 2016 Mar 2 [cited 2016 Mar 29]; Disponible en: http://www.elglobal.net/ noticias-medicamento/2015-02-27/ farmacia/la-aemps-intensifica-el-cierre-de-webs-ilegales-que-venden-farmacos/pagina.aspx?idart $=896048$

32. Colegio Oficial de Farmacéuticos de Madrid. La AEMPS lanzará una nueva campaña de información para alertar de los riesgos de compra de fármacos en la red fuera de las farmacias autorizadas. COFM, Noticias [Internet]. Madrid; 2016 Mar 10; Disponible en: http:// www.cofm.es/es/comunicacion/ noticias/belen-escribano-el-farmaceutico-juega-un-papel-clave-para-que-no-lleguen-medicamentos-falsificados-a-los-pacientes. $\mathrm{html}$ ? idCategoria $=1 \mathrm{ftech}$ fes $\mathrm{de}=$ Ctexto $=$ EtfechaHasta $=$

33. Agencia Española de Medicamentos y Productos Sanitarios, Ministerio de Sanidad Servicios Sociales e Igualdad, Gobierno de España. Estrategia frente a medicamentos falsificados 2016-2019. [Internet]. AEMPS; 2016. Disponible en: https://www.aemps. gob.es/publicaciones/publica/docs/ Estrategia_falsificados_2016-2019. pdf

34. Agencia Española de Medicamentos y Productos Sanitarios y Ministerio de Sanidad Servicios Sociales e Igualdad. Riesgos de la compra de medicamentos en web ilegales [Internet]. Riesgos de la compra de medicamentos en web ilegales. 2016. Disponible en: https://distafarma.aemps.es/ farmacom/faces/templates/estaticas/ riesgos.xhtml

35. European Union. fakeshare.eu [Internet]. Disponible en: http://www. fakeshare.eu/es

36. Agencia Española de Medicamentos y Productos Sanitarios. Distafarma. Campaña informativa [Internet]. Available from: https://distafarma. aemps.es/farmacom/faces/templates/ estaticas/campania.xhtml

37. El farmaceutico.es. Belén Escribano: "El farmacéutico juega un papel clave para que no lleguen medicamentos falsificados a los pacientes». elfarmaceutico.es, Crónicas, Infarma 2016 [Internet]. 2016 Mar 10; Disponible en: http://elfarmaceutico.es/index. $\mathrm{php} /$ noticias-actualidad-farmaceutica/item/6865-belen-escribano-el-farmaceutico-juega-un-papel-clave-para-que-no-lleguen-medicamentos-falsificados-a-los-pacientes\#. Vvq2TuKLTIU 